\title{
The conditioned suppression and enhancement of avoidance during a serial compound CS
}

\author{
ALBERT E. ROBERTS \\ Catawba College, Salisbury, North Carolina 28144 \\ and \\ H. M. B. HURWITZ \\ University of Guelph, Guelph, Ontario, Canada
}

\begin{abstract}
Twelve rats were trained under a free operant avoidance schedule. Then a signal S1) followed by a shock (US) was superimposed on avoidance behavior. Later, a second signal (S2) was interposed between S1 and US. The rats were divided into two groups and, in addition to S1-S2-US, received either S1 or S2 singly. Finally, the S1-S2 sequence was given without US. Suppression of avoidance developed under the S1-US procedure, but when S1 was separated from US by S2, suppression gave way to enhancement in avoidance during S1. Moreover, when S2 was presented independently of the S1-S2-US sequence, the suppression of avoidance diminished during S2, although differential avoidance behavior during the sequences was unaffected. Finally, when S1-S2 was presented without US, suppressed avoidance during S2 disappeared, while avoidance enhancement during $\mathrm{S} 1$ was maintained. The results were interpreted in terms of the necessity of the CS being a reliable predictor of an imminent US for the maintenance of conditioned avoidance suppression.
\end{abstract}

Over the past several years, the widely held view that Pavlovian and operant learning involve fundamentally different and independent processes has undergone modification. For example, operant behavior can be rich in Pavlovian learning (e.g., autoshaping), while certain Pavlovian phenomena can be brought under operant control (e.g., biofeedback). The study of the mutual influence between operant and Pavlovian procedures is more commonly known as an "operant-Pavlovian interaction." One dimension of this interaction centers on the superimposition of Pavlovian (CS-US) training, using an aversive US, upon an ongoing operant response rate; changes in the response rate during the CS are attributed to the Pavlovian procedure. An early example is found in the study by Estes and Skinner (1941), who reported that food-maintained responding diminished during a stimulus (CS) that was followed by a shock-US, that is, conditioned suppression.

When the interaction involves avoidance-motivated behavior, the effects of the CS-US training are more difficult to determine (cf. Hurwitz \& Roberts, 1977). The avoidance procedure most widely used involves an electric shock occurring at regular intervals unless a criterion response occurs, in which case the shock is postponed for a fixed period of time (Sidman, 1953). The literature indicates that while avoidance often is suppressed under CS-US training, a number of parameters can affect the development of a suppressive effect by the CS; these have been reviewed and discussed elsewhere (cf. Hurwitz \& Roberts, 1977). However, we can point to the maintenance of the CS-US association as a prime condition not only for the development but also for the continuation of suppressed operant avoidance. That is, when the US subsequently is removed (i.e., Pavlovian extinction), an enhancement in avoidance during the CS often is observed (Hurwitz \& Roberts, 1971; Roberts \& Porter, 1976).

Several questions about the nature of the CS-US association remain unanswered. For example, the literature indicates that the CS must "reliably" predict the US for the CS to acquire a suppressive influence (cf. Rescorla, 1967). Even though the CS is a reliable predictor of the US, conditioned avoidance suppression occurs only when the CS-US delay interval is short and not when the delay interval is long (Shimoff, 1972). The temporal aspect of CS-US consequently appears critically important, as the CS apparently must predict an imminent US to suppress avoidance. The present experiment studied this view by separating CS and US by a delay interval rather than removing the US altogether. A second stimulus was inserted between the CS and US to identify the delay period. The use of two sequential stimuli (S1 and S2) also enabled us to study the contribution of reliable prediction of a CS in maintaining conditioned avoidance suppression. That is, the three stimuli (the original CS, noted S1, the new stimulus, S2, and the shock-US) continued to be given in the sequence, but S1 occasionally was presented singly to one group, while S2 was presented singly to a second group. Under this arrangement, $\mathrm{S} 1$ became an unreliable predictor of US to the first group as it only sometimes was followed by $\mathrm{S} 2$ and the US. On the 
other hand, S1 continued to reliably predict US to the second group because S2 sometimes was given alone. In this case, $\mathrm{S} 2$ alone predicted the nonoccurrence of US. We could determine with these procedures if the control over avoidance acquired by a stimulus given in a sequence terminating with a shock-US would alter or be unchanged by its being given singly.

\section{METHOD}

Subjects

The subjects were 12 female hooded rats, purchased from Blue Spruce Farms, New York, weighing about $175 \mathrm{~g}$ at the beginning of the experiment. The rats were housed in the main animal vivarium with food and water always available.

\section{Apparatus}

Three $23 \times 24 \times 14 \mathrm{~cm}$ metal leverpressing chambers were used, each with a $5-\mathrm{cm}$ lever protruding $2.5 \mathrm{~cm}$ into the chamber $5 \mathrm{~cm}$ above the grid floor. A $10 \mathrm{~g}$ deadweight $(.1 \mathrm{~N})$ on the lever activated the microswitch. The grids consisted of $.25-\mathrm{cm}$ brass rods spaced $1.3 \mathrm{~cm}$ apart and parallel to the width of the lever. Two ceiling houselights provided illumination. Scrambled shock was delivered to the grids, lever, and sides of the chamber. Each chamber was placed in a larger sound-insulated and ventilated box. A relay attached to the outside rear wall of each chamber provided a clicking signal (S1) at 10 clicks/sec and added $10 \mathrm{~dB}$ to the resting noise level $(78 \mathrm{~dB})$. S2 was a tone $(2,800 \mathrm{~Hz})$ (Sonalert) that added $11 \mathrm{~dB}$ to the resting noise level.

\section{Procedure}

First, the rats received $392-\mathrm{h}$ avoidance training sessions. Under the avoidance schedule used, $1.1-\mathrm{sec} .8-\mathrm{mA}$ shock was delivered every $5 \mathrm{sec}$ unless a leverpress occurred. Each leverpress briefly $(.2 \mathrm{sec})$ turned off the houselights and postponed shock for $15 \mathrm{sec}$. This avoidance schedule remained in effect throughout the experiment. Second, for 15 sessions, Pavlovian training was superimposed on the avoidance baseline. S1 was given for $60 \mathrm{sec}$ and was immediately followed by a 1-sec .8-mA shock-US on the average of once every $6 \mathrm{~min}$. In the third phase (30 sessions), the 60-sec warning period was divided so that the original stimulus (S1) was given for $30 \mathrm{sec}$ and was followed by a new stimulus (S2) for $30 \mathrm{sec}$; the US occurred at the end of S2. During the fourth phase (30 sessions), two groups were arranged so that the rates of unavoided shock were comparable. Both groups continued to receive the S1-S2-US sequence (20 times/session), but Group $1 \quad(n=6)$ also was given S1 alone 10 times/session. Group $2(n=6)$ was given $S 2$ alone 10 times/session; on these occasions, S2 was neither preceded by $\mathrm{S} 1$ nor followed by US. The fifth phase was identical to the fourth except that the US no longer was given (i.e., Pavlovian extinction). Groups 1 and 2 received 30 and 20 sessions, respectively, under this phase. Finally, all stimuli were withdrawn and 10 avoidance sessions were given (i.e., a replication of the procedure of the first phase).

Changes in avoidance behavior were assessed by recording both response and shock frequencies during S1 and S2 and in the 60 -sec period prior to the signals (baseline). Mean data were calculated for each subject over the final four sessions of each phase, and these means provided the bases for statistical analyses.

\section{RESULTS}

Figure 1 gives mean response and shocks per minute, in blocks of 2 sessions, for the final 10 avoidance training sessions and all S1-US and S1-S2-US sessions.

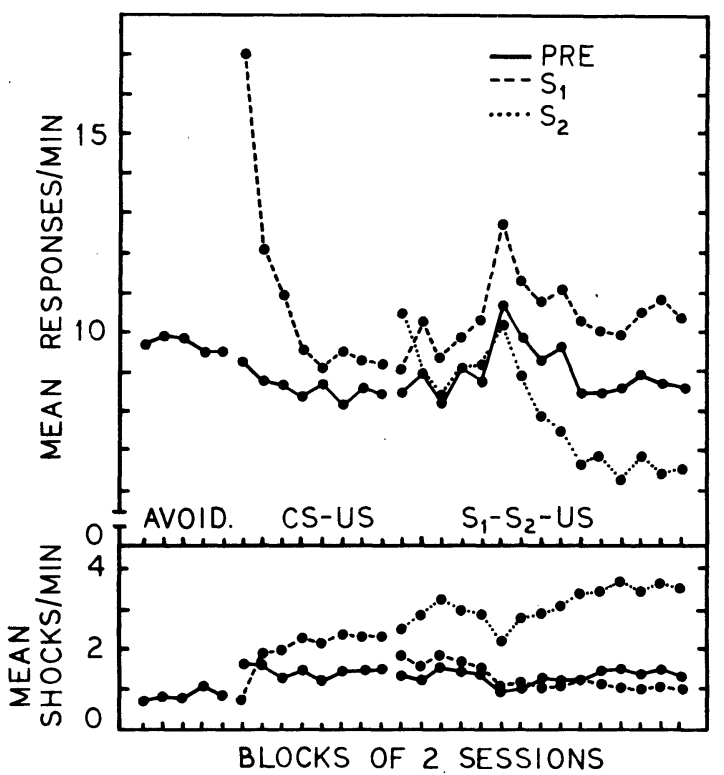

Figure 1. Mean response rates (upper panel) and shock rates (lower panel) for the final avoidance training (Phase 1), S1-US (Phase 2), and S1-S2-US (Phase 3) sessions. Note-The first data points of S1-US are for a single session.

Figure 1 shows that both mean response and shock rates were stable over the final sessions of training.

\section{Phases 2 and 3: S1-US and S1-S2-US}

Response rate during S1 was almost $100 \%$ above the baseline rate in the first Phase 2 (S1-US) session, while the shock rate was less than baseline. By the end of Phase 2, direct difference $t$ tests indicated that shock rate in $\mathrm{S} 1$ was reliably greater than baseline $[t(11)=3.70, p<.01]$, while response rate differences between baseline and $S 1$ were unreliable $(t<1)$. The reader should note that compared to Phase 1 , baseline response rate was reduced $[\mathrm{t}(11)=2.49, \mathrm{p}<.05]$ and baseline shock rate increased $[\mathrm{t}(11)=2.53, \mathrm{p}<.05]$ under Pavlovian training. Figure 1 shows that inserting S2 (Phase 3) did not change either the baseline response or shock rate from Phase 2 levels. The effect of the compound CS on avoidance measures was analyzed by repeated-measures ANOVAs, with Tukey tests comparing stimulus conditions when warranted. All differences in measures were unreliable in the first session of Phase 3. But by the end of this phase, the two stimuli assumed differential control over response rate $[F(2,22)=15.46, p<.001]$ as responding was increased during $\mathrm{S} 1(\mathrm{p}<.001)$ and reduced in $\mathrm{S} 2(\mathrm{p}<.05)$, each compared to baseline. Shock rate differences also were reliable $[F(2,22)=53.99, p<.001]$ as the shock rate in $\mathrm{S} 2$ was increased over both baseline and S1 (ps $<.001$ ); differences between baseline and S1 shock rates were not reliable.

\footnotetext{
"Reliable Prediction": S1 and S2 Given Singly
}

In Phase 4 the S1-S2-US sequence continued to be 
given to each of two groups, but S1 occasionally was given alone to Group 1 and $\mathrm{S} 2$ was given alone to Group 2. In Phase 5 the US was withdrawn for both groups, and Phase 6 continued avoidance training but without the stimuli. Group differences on all avoidance measures based on the terminal performances under Phase 3 were statistically unreliable. Figure 2 gives mean response and shock rates over the final six sessions of Phase 3 and for the remainder of the experiment for Group 1 (left-side panel) and Group 2 (right-side panel). Figure 2 shows, and ANOVAs confirm, that presenting the individual stimuli singly did not alter the differential control of those stimuli over the response and shock rates that had developed in Phase 3. Response rate was elevated in S1 periods and reduced in S2 periods, compared to baseline, while shock rate was reduced in $\mathrm{S} 1$ and elevated in S2. Not surprisingly, the avoidance performance of Group 1 during the two S1 conditions was nearly identical. However, in contrast to the avoidance impairment during S2 when S2 was part of the compound $\mathrm{CS}$, response and shock rate differences between $\mathrm{S} 2$ alone and baseline had become unreliable by the end of Phase 4 (see Group 2). When the US was removed in Phase 5, responding increased and shock rate lessened in all periods for both groups. But S1 continued to control and increase response rate and a reduced shock rate compared both to baseline and $\mathrm{S} 2$; differences between the latter two periods had become unreliable by the end of Phase 5. In avoidance retraining (Phase 6), the response rate of Group 1 did not change reliably while baseline shock rate increased, compared to Phase $5[\mathrm{t}(5)=4.02, \mathrm{p}<.02]$. Baseline measures were not reliably changed in Phase 6 for Group 2 .

\section{DISCUSSION}

A preshock stimulus presented on an avoidance baseline will, after many presentations, control a deterioration in avoidance (see review by Hurwitz \& Roberts, 1977). The purpose of the present experiment was to determine if the maintenance of conditioned avoidance suppression depended on the temporal contiguity between the CS and US. The question was investigated by interposing a second stimulus between the CS and US so as to form a sequence of S1-S2-US. The results showed that under such an arrangement, S1 lost its acquired suppressive function and developed just the opposite function: S1 served to enhance avoidance. This change in control found for S1 is similar to that typically found under CS extinction, that is, presenting the CS with the US omitted.

The second stimulus in the sequence (S2) acquired suppressive control over avoidance when S2 appeared as part of that sequence; but this suppressive control extinguished when S2 was given independently of the sequence (Group 2, Phase 4). However, the suppressive influence found for $S 2$ in the stimulus sequence also extinguished when the US was removed in Phase 5 (although, in contrast to S1, an enhancement capability did not develop). These data support a view that a CS must predict an imminent US for conditioned avoidance suppression to be maintained (for a similar view, see Seger \& Scheuer, 1977).

Our experiment did not use an unsignaled trace conditioning procedure (i.e., S1-gap-US) so the post-S1 period (the gap) would not be confused with other unsignaled periods of the session. This leads us to speculate on the degree to which the differential changes in avoidance depended on the presence of the two stimuli in the preshock sequence. In this regard, data presented, by Shimoff (1972) are helpful. In the Shimoff procedure, separate groups of subjects were trained under one of several durations of a tone CS, and an unsignaled trace interval
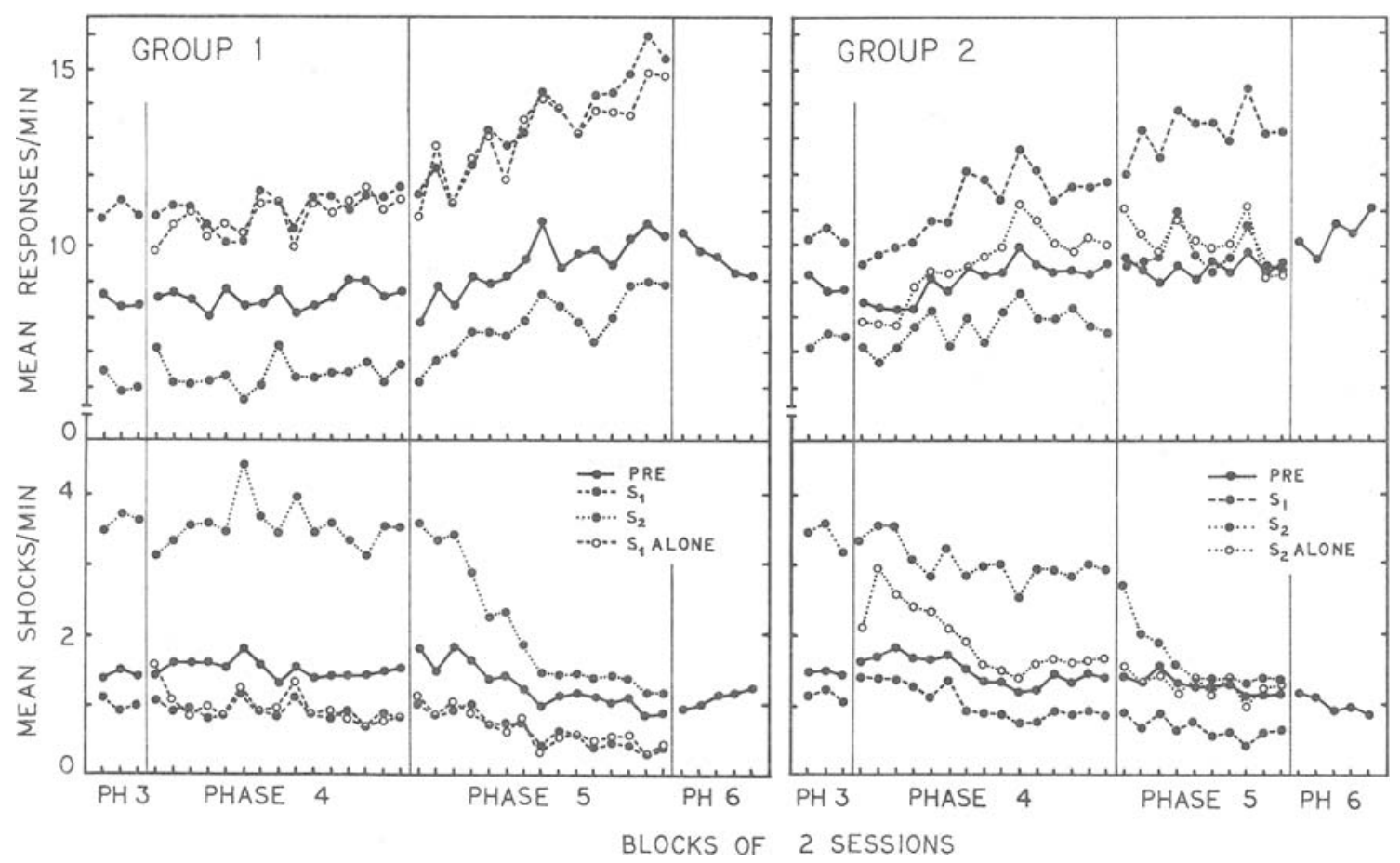

Figure 2. Mean response and shock rates for the two groups obtained from the final phases of the experiment (see text for details). 
was varied. Shimoff found that response rate during the trace interval came to match baseline as the interval was increased; for example, avoidance suppression was not found during the gap. However, avoidance enhancement was not found during the CS under the trace conditioning procedure. The training procedures between the two experiments differ in that Shimoff trained his subjects under a given trace interval from the outset, whereas we separated S1 and US after they had been contiguously associated. With the Shimoff data as points of reference, S2 seemed to do more than simply identify the trace interval. Rather, by being contiguously associated with US (in Phase 3), S2 acquired a suppressive capability (as S1 did during Phase 2), while S1 lost suppressive control (and acquired enhancement control).

The notion that the change in influence of $\mathrm{S} 1$, from suppression to enhancement, was related to the dissociation between S1 and US in Phase 3 is given credence by the decrease in suppressive control of S2 (1) when given singly in Phase 4, and (2) when US did not follow the stimulus sequence in Phase 5. But our finding that avoidance was enhanced during $\mathrm{S} 1$, rather than coming to approximate baseline values, was both unexpected and puzzling, particularly since a similar outcome was not obtained with S2. Three possible explanations for the enhancement influence shown by $\mathrm{S} 1$ can be offered. First, the outcomes obtained for S1 (Phase 3) and S2 (Phase 5) actually may reflect a "loss in conditioned effect." That is, S1 may have had an enhancement influence, and S2 minimal influence, over the avoidance baseline prior to the conditioning phases. The extinction data that were obtained may reflect a return to these preconditioning influences. As neither stimulus was offered in pretest, this possibility cannot be evaluated.

A second possibility centers on the relationship between S1 and US. When the two were separated in Phase 3, S1 could have become a "safety" signal by indicating that the US was not imminent. Occasionally giving $S 1$ singly in Phase 4, then, would underscore the safety message. Removing US at the end to the stimulus sequence in Phase 5 would not alter the prediction of "safety" by S1. The third possibility gives attention to the S1-S2 association. Since S1 was followed by S2 in Phase 3, rather than by US, S1 could have become a conditioned inhibitor (and would produce a change in avoidance opposite to that as a conditioned excitor in Phase 2). That is, the enhancement control of S1 was maintained (i.e., reinforced) by the occurrence of S2. Since the experiment was designed to address another purpose, these possibilities cannot be evaluated directly.

Returning to the major results of the experiment, the data suggest that a simple "predictive association" between a CS and a US is not a sufficient condition for a preshock CS to maintain conditioned avoidance suppression. Rather, the CS must reliably predict the imminent occurrence of US. Even though a CS had been paired with the US, conditioned avoidance suppression during that CS was not maintained in three instances: (1) when a new stimulus was interposed between that CS and US, (2) when the CS was given alone and, thus, negatively correlated with US, and (3) when the US was removed altogether.

\section{REFERENCES}

Estes, W. K., \& Skinner, B. F. Some quantitative properties of anxiety. Journal of Experimental Psychology, 1941, 29, 390-400.

Hurwitz, H. M. B., \& Roberts, A. E. Conditioned suppression of an avoidance response. Journal of the Experimental Analysis of Behavior, 1971, 16, 275-281.

Hurwitz, H. M. B., \& Roberts, A. E. Aversively controlled behavior and the analysis of conditioned suppression. In $\mathrm{H}$. Davis \& H. M. B. Hurwitz (Eds.), Operant-Pavlovian interactions. Hillsdale, N.J: Erlbaum, 1977.

Rescorla, R. A. Pavlovian conditioning and its proper control procedures. Psychological Review, 1967, 74, 71-80.

Roberts, A. E., \& Porter, J. W. CS-US training, method of baseline shock delivery, and the conditioned suppression of shuttle avoidance. Psychological Record, 1976, 26, 377-386.

Seger, K. A., \& Scheuer, C. The informational properties of $\mathrm{S} 1, \mathrm{~S} 2$, and the $\mathrm{S} 1-\mathrm{S} 2$ sequence on conditioned suppression. Animal Learning \& Behavior, 1977, 5, 39-41.

Shimoff, E. Avoidance responding as a function of stimulus duration and relation to free shock. Journal of the Experimental Analysis of Behavior, 1972, 17, 451-461.

Sidman, M. Avoidance conditioning with brief shock and no exteroceptive warning signal. Science, 1953, 118, 157-158.

(Received for publication April 19, 1979.) 